\title{
Cuidar la Salud Bucal es una cuestión de actitud
}

Sofía de los Milagros Alí

Profesora Adjunta. Cátedra Odontología Social y Comunitaria.

\section{Roxana Lilian Blanco}

Auxiliar Docente de Primera Categoría. Cátedra Odontología Social y Comunitaria.

María Alejandra Meana Auxiliar Docente de Primera Categoría. Cátedra Odontología Social y Comunitaria.

\section{Resumen}

El problema odontológico es uno de los problemas que afectan a un alto porcentaje de las personas con discapacidad. Objetivos del trabajo, determinar el estado de salud bucal de estas personas, como así también instruir a recursos humanos en la atención de las mismas y estimular la intervención y compromiso de los padres y tutores de los pacientes en el cuidado de la salud bucal. Por la magnitud del problema identificado, consideramos, que la medida más eficaz y prioritaria para reducir la prevalencia e incidencia de enfermedades bucales, es la Promoción de la Salud, la cual proporciona los medios necesarios para mejorar la salud y ejercer un mayor control sobre la misma y la Prevención, orientada a evitar, la aparición de las secuelas. Además se trata de lograr un nexo con la Facultad de Odontología a través de la Cátedra Odontología Social y Comunitaria, con el fin de satisfacer las demandas encontradas.

\section{Palabras Clave}

Personas, Discapacidad, Salud Bucal.

\section{Summary}

The dental problem is one of the problems affecting a high percentage of people with disabilities. Objectives of work, determine the oral health status of these people, as well as instruct human resources in the care of them and to stimulate the involvement and commitment 
of parents and guardians of patients in the care of oral health. Because of the magnitude of the problem identified, we believe that the most effective and priority to reduce the prevalence and incidence of oral diseases measure, is the Health Promotion, which provides the means to improve health and exercise greater control over it and Prevention, aimed at avoiding the emergence of the consequence. In addition it is to achieve a link with the Faculty of Dentistry through the Chair Social and Community Dentistry, in order to meet the demands found.

\section{Keywords}

People, Disability, Dental Health.

\section{Resumo}

O problema odontológico é um dos problemas que afetam a uma alta percentagem das pessoas com discapacidade. Objetivos do trabalho, determinar o estado de saúde bucal destas pessoas, como assim também instruir a recursos humanos na atenção das mesmas e estimular a intervenção e compromisso dos pais e tutores dos pacientes no cuidado da saúde bucal. Pela magnitude do problema identificado, consideramos, que a medida mais eficaz e prioritária para reduzir a prevalencia e incidência de doenças bucales, é a Promoção da Saúde, a qual proporciona os meios necessários para melhorar a saúde e exercer um maior controle sobre a mesma e a Prevenção, orientada a evitar, o aparecimento das secuelas. . Ademais trata-se de conseguir um nexo com a Faculdade de Odontología através da Cátedra Odontología Social e Comunitária, com o fim de satisfazer as demandas encontradas.

\section{Palavras chave}

Pessoas, Discapacidade, Saúde Bucal.

\section{Introducción}

La salud es un derecho de todos los ciudadanos, y al mismo tiempo un gran desafío para los pueblos, por la complejidad y resistencia que todo cambio genera. El análisis de la equidad en salud, es complejo y en muchas ocasiones la discriminación se refleja en aquellos que no logran acceder a la calidad de asistencia y de prestaciones debido a su condición. Un ejemplo, se observa en la calidad de salud bucal, de las personas con discapacidad.

La causa de una desigualdad, como dice Whitehead (I99I)', tiene que ser examinada en el contexto de lo que sucede en el resto de la sociedad. Luego, el codificar una desigualdad, como injusta, innecesaria y/o evitable o no, depende de un valor de juicio.

La salud bucal es parte de la salud general y como tal debe expresarse al considerar las necesidades de las personas, es decir, involucra al paciente como una unidad armónica, íntimamente unido a su familia o a quien se ocupa de él, además adaptado a su ambiente geográfico, social, cultural, y económico. La salud humana está también amenazada en las dimensiones psicológicas y sociales, debido a los ritmos de vida: estrés, relaciones humanas, desarraigo, exclusión social, entre otras ${ }^{2}$.

La concepción del fomento de la salud bucal debe dirigirse hacia dos vertientes fundamentalmente: una dirigida a controlar los factores determinantes y otra a identificar a los individuos susceptibles de riesgo para ofrecerle protección individual.

Los determinantes que condicionan la salud general también afectan e influyen sobre la salud oral. Estos se pueden definir como los factores, condiciones o circunstancias que influyen en el nivel de salud de los individuos y poblaciones.

En la actualidad la salud y la calidad de vida tienen un espacio central en nuestra sociedad. La misma es un derecho, un valor, una aspiración, un recurso, una necesidad, una demanda social comprendida en el centro de las decisiones en el ámbito público y privado. Los determinantes sociales como lo son la situación económica, la educación, la ocupación, el lugar donde viven y trabajan, impactan en la salud y enfermedad de las personas, más aun cuando se trata de personas con discapacidad. Tovar y García citados por Velez ${ }^{3}$ manifiestan que los determinantes sociales en salud comprenden las condiciones en que las personas nacen, crecen, viven, trabajan y envejecen, así como las desigualdades que se manifiestan en estas condiciones también crean desigualdades en salud. Es importante hacer hincapié en los determinantes sociales de salud, lo 
cual permite hacer un abordaje de una estrategia que busca aplicar el conocimiento científico acumulado en relación a las causas últimas o estructuras de los problemas de salud y de recuperar estrategias de salud para todos ${ }^{4}$.

Teniendo en cuenta lo antes mencionado, se sabe que la incidencia y las consecuencias de la discapacidad se encuentran estrechamente relacionadas con factores sociales y que se presentan con mayor frecuencia en zonas de bajos ingresos y de bajo nivel educativo al tener mayor exposición a diversos factores de riesgo. Las razones por las que las personas presentan enfermedades que afectan la salud bucal son múltiples y complejas, pues se debe a la interacción de diversos factores de riesgo que a su vez se ven afectados por los diversos determinantes sociales de la salud y a su situación de discapacidad.

En muchos estudios realizados en Europa ${ }^{5}$, también se han encontrado que el estado de salud bucodental es diferente según el tipo de discapacidad que presente la persona. Es así, que han indicado que las personas con discapacidad intelectual presentan más problemas periodontales, mayor número de caries sin tratar, dolores orales más frecuentes y más piezas dentales perdidas en comparación con las personas sin discapacidad intelectual. Así mismo, Dávila, María E y colaboradores 6 , realizaron, en Venezuela, una investigación descriptiva transversal, con el propósito de determinar la prevalencia de caries dental en personas con retraso mental (RM) y Síndrome de Down (SD). Las personas con RM moderado presentaron el mayor porcentaje de caries dental.

Un estudio que se realizó en Australia sobre la atención odontológica de los niños con discapacidades, se observó que las necesidades de tratamientos sencillos del $41 \%$ de la muestra no estaban satisfechas ${ }^{7}$. Zamora realizó un estudio transversal, comparativo en la ciudad de Córdoba, Argentina en el que participaron 132 escolares con discapacidad motora y retraso intelectual asociado de 5 a 13 años de edad ${ }^{8}$.

Una investigación realizada por Navas y $\mathrm{col}^{9}$ reportó que los padres de niños con síndrome de Down manifestaron que frecuentemente encontraban problemas para tener acceso a la atención de salud bucal de sus hijos, y que estos pacientes tenían menos probabilidades de recibir servicios odontológicos que aquellos individuos sin discapacidad.

La mayoría de los pacientes con discapacidad, requieren de la supervisión de los padres o de las personas que los asisten, el odontólogo debe percibir la problemática del individuo. Y también transmitirles a los padres y al paciente como debe realizarse una buena la higiene bucal ${ }^{10}$.

\section{Materiales y Métodos}

Se realizó un estudio de diseño epidemiológico transversal, descriptivo y de intervención. La población de estudio: Personas con Discapacidad Intelectual que concurran al Servicio de Atención Odontológico para Pacientes con Capacidades Diferentes de la FOUNNE y el Instituto Crecer con Todos.

Se trabajó sobre una muestra de 100 personas con discapacidad intelectual (Clasificación Internacional del Funcionamiento de la Discapacidad y de la Salud - CIF)

Criterios de Inclusión: Personas con Discapacidad Intelectual de ambos sexos, con o sin acompañamiento de tutor encargado o responsable, sin límite de edad que concurren al Servicio Odontológico para Pacientes con Capacidades Diferentes de la FOUNNE.y el Instituto Crecer con Todos, con certificado de Discapacidad e historia clínica, procedentes de la provincia de Corrientes.

\section{Criterios de Exclusión:}

Se excluirán de la muestra: Personas con Discapacidad motora y sensorial.

\section{Parámetros a Evaluar:}

En las personas: Categorización de la discapacidad mental: leve - moderada- grave

\section{A las familias:}

Información de aspectos socioculturales: Conductas del tutor hacia el cuidado de la salud bucal.

En los Servicios: Tipos de prestaciones y cobertura a la población con discapacidad.

Las actividades realizadas se registraron en historias clínicas de la FOUNNE, sobre el estado del componente bucal de la salud y Consentimiento Informado por el padre, tutor o responsable del paciente, aprobado por el Comité de Bioética de la FOUNNE. También se realizaron encuestas estructuradas a la familia sobre datos socio-culturales. 


\section{Resultados parciales}

Los distintos tipos de deficiencias registradas en los pacientes con discapacidad intelectual encuestados se detallan a continuación:

Tabla $N^{\circ}$. Tipo de deficiencias en pacientes con discapacidad intelectual que asisten al Instituto Crecer con Todos y al Servicio Odontológico para Pacientes con Capacidades Diferentes de la FOUNNE.

\section{Tipo de Deficiencias}

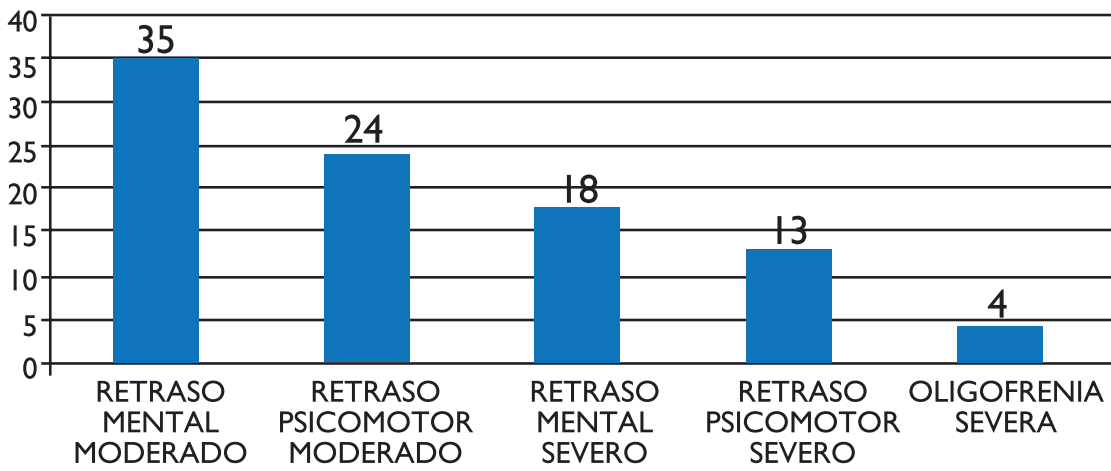

También se obtuvieron datos en cuanto al tipo de prestaciones que recibieron anteriormente estas personas.

Tabla $\mathbf{N}^{\circ} 2$. Porcentaje según tipo de prestaciones odontológicas de personas con discapacidad intelectual concurrentes al Instituto Crecer con Todos, y al Servicio Odontológico para Pacientes con Capacidades Diferentes de la FOUNNE.

\begin{tabular}{lc}
\hline Tipo de Prestaciones & Porcentaje \\
\hline Sin Atención & 41 \\
\hline Preventiva & 5 \\
\hline Conservadora-Rehabilitadora & 8 \\
\hline Extraccionista & 46 \\
\hline Totales & 100 \\
\hline
\end{tabular}

De las encuestas realizadas también se pudo obtener los siguientes resultados en cuanto a la conducta que adoptan padres, tutores o encargados ante el cuidado de la salud bucal de las personas con discapacidad.
Gráfico $N^{\circ}$ I. Porcentaje según conducta que adoptan padres, tutores o encargados ante el cuidado de la salud bucal de las personas con discapacidad, concurrentes al Instituto Crecer con Todos, y al Servicio Odontológico para Pacientes con Capacidades Diferentes de la FOUNNE.

Conductas de los padres, tutores o encargados de las personas con discapacidad intelectual

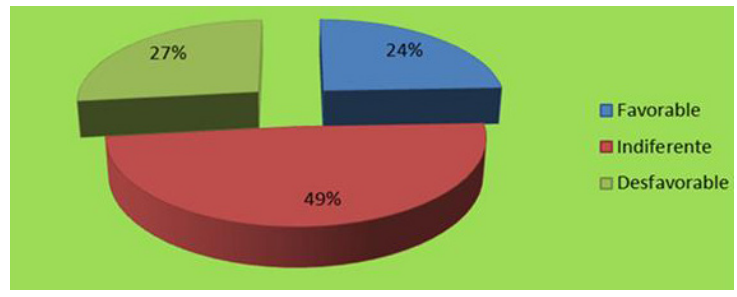


REVISTA FACULTAD DE ODONTOLOGÍA

ISSN No 1668-7280 - Vol. IX No 1 - 2016

33
INVESTIGACIÓN

Cuidar la Salud Bucal es una cuestión

de actitud

\section{Discusión}

Si partimos de la definición ya mencionada por la Convención Internacional sobre los Derechos de las Personas con Discapacidad", en la segunda parte del artículo I, "Las personas con discapacidad incluyen a aquellas que tengan deficiencias físicas, mentales, intelectuales o sensoriales a largo plazo que, al interactuar con diversas barreras, puedan impedir su participación plena y efectiva en la sociedad, en igualdad de condiciones con las demás", podemos ver, que de esta definición surge como se puede apreciar; que hablamos de personas con discapacidad las que se ven impedidos de participar plenamente en su vida cotidiana. Así mismo se habla de barreras, las mismas nos puede hacer pensar, en algo arquitectónico, físico. Pero en realidad esas barreras son establecidas, edificadas y proyectadas por la misma sociedad. Teniendo en cuenta esto, podemos decir que la discapacidad depende de cómo está diseñada y funcionan nuestras estructuras sociales.

En concordancia con el estudio realizado por Zamora ${ }^{8}$, a través de programas de intervención, clínica-educativa permite observan indicadores más compatibles con la salud que aquellos escolares que no han recibido dicha intervención.

El manejo de las personas con discapacidad en el ámbito odontológico reviste una especial idiosincrasia que conduce a plantearse algunas cuestiones complejas, como por ejemplo el derecho de las personas con discapacidad intelectual, a decidir que sus dientes tengan o no caries, o si quieren recibir o no cuidados odontológicos. En cualquier caso los profesionales de la salud oral y los cuidadores de estos pacientes, tienen el deber de procurar garantizarles una boca saludable, lo que exige una formación específica que les proporcione conocimientos y habilidades que potencien el concepto de los "cuidados orales preventivos"'2.

\section{Conclusión}

Las personas con discapacidad son identificadas como colectivo vulnerable a sufrir algún tipo de exclusión. Es así que en el Informe Mundial sobre la discapacidad (OMS 20I I) ${ }^{13}$ se puso de manifiesto los principales obstáculos para este grupo.

Debido al problema identificado, consideramos, para reducir la prevalencia e incidencia de enfermedades bucales, la medida más eficaz y prioritaria es la Promoción de la Salud, la cual proporciona los medios necesarios para mejorar la salud y ejercer un mayor control sobre la misma y la Prevención, la cual consiste en medidas orientadas a evitar, la aparición de las secuelas.

El astrofísico británico Stephen Hawking escribió un prólogo en el Informe mundial sobre la discapacidad, publicado por la Organización Mundial de la Salud (OMS) y el Banco Mundial, en Ginebra en $20 \mathrm{I} \mathrm{I}^{14}$, que arranca con esta consideración contundente: La discapacidad no debería ser un obstáculo para el éxito. El estado negativo de salud, la deficiencia o daño de la persona afectada explica sólo una parte de la condición y de la situación de discapacidad. Por ello, se necesita tanto una adecuada rehabilitación como contar con una sociedad accesible ${ }^{15}$. 


\section{Bibliografía}

I. Whitehead M, Dahlgren G. Levelling. Concepts and principles for tackling social inequities in health. Studies on social and economic determinants of population health no. 2 [serie en Internet]. 2007. Disponible en: http://www.euro.who. int/document/e89383.pdf

2. Palomino Moral, P., Grande Gascón, M., Abad, M. La Salud y sus Determinantes Sociales. Desigualdades y exclusión en la sociedad del siglo XXI. Revista Internacional de Sociología (RIS), [Serie en Internet] Jun 20I4. Vol. 72, extra I 7I-9I. Disponible en: http//www.apsredes.org/site2013/ wp-content/.../2014/.../laSaludYSusDeterminantes

3. Vélez A., Vidarte C., Cerón B. Determinantes sociales de la salud y discapacidad: caso Santiago de Cali. Rev. Fac. Nac. Salud Pública [Serie en Internet]. 2013 Dic 31( Supl I ): 192-202. Disponible en: http://www.scielo.org. co/scielo.php?script=sci_arttext\&pid=SOI 20 386X20I300040002 I\&Ing=en.

4. Álvarez LS. Los determinantes sociales de la salud: Más allá de los factores de riesgo. Rev. Gerenc. Polit. Salud. Bogotá (Colombia), [Serie en Internet]. 20II. Disponible en: www.scielo.org. co/pdf/rgps/v8n I7/v8n I7a05.pdf

5. Tamarit Cuadrado, J; Muñoz Bravo J.; Maeso Martínez, P.; Belinchón Carmona, M. Indicadores de Salud en Personas con Discapacidad Intelectual. Edita: FEAPS. Confederación Española de Organizaciones en favor de las Personas con Discapacidad Intelectual. Madrid, 2010.

6. Dávila, ME., Gil, M., Daza, D., Bullones, X., Ugel, E.. Caries dental en las Personas Con Retraso mental, Síndrome de Down y. Rev. Salud Pública [serie en Internet]. 2006 Dic [Citado el 28 de Agos 20I I], 8(3):207-2I3. Disponible en: http:// www.scielosp.org/scielo.php.

7. Universidad Nacional Autónoma de Honduras Facultad de Ciencias Médicas. Situación Mundial de la Discapacidad. OPS. Marzo 2013.

8. Zamora, I., Cornejo, L. Salud Bucal de Escolares con Discapacidad Motora y Retraso Intelectual asociado. Revista de vinculación con la comunidad de la Faculta de Odontología de la Universidad Nacional de Córdoba. [Serie en Internet]. 20 I4. Disponible en: http://www.dialnet.unirioja. es/descarga/articulo/4838329.pdf

9. Navas P Rita, Mogollón Joalis. Participación de los padres en el cuidado de la salud bucal de los niños y adolescentes con síndrome de Down. Ciencia Odontológica [revista en la Internet]. 2007 Dic; 4(2): 130-140. Disponible en: http://www2.scielo. org.ve/scielo.php?script=sci_arttext\&pid=SI 3 I782452007000200004\&lng=es.

10. Córdoba Urbano, D., España Coral, S., Tapia T., G. Conocimientos y actitud de los odontólogos en la atención de personas con discapacidad intelectual. Rev Univ. Salud. [Serie en Internet]. 2012 Ene [Citado 27 de Ene 2014] ; 14(I): 78-86. Disponible en: http://www.scielo. org.co/scielo.php?script=sci_arttext\&pid=SO I 24710720I2000100007\&Ing=en

II. Convención Internacional sobre los Derechos de las Personas con Discapacidad. Naciones Unidas [serie en Internet]. (2007) Disponible en: http:// www.sidar.org/recur/direc/legis/convencion.php

12. Serrano, María Esther Pérez, Limeres Posse, Jacobo, Fernández Feijoo, Javier. Manual de Higiene Oral para Personas con Discapacidad. Disponible en: www.seoeme.es/media/uploads/ manual_higiene_oral.pdf

13. Presidencia de la Nación. Consejo Nacional de Coordinación de Políticas Sociales. Comisión Nacional Asesora para la Integración de Personas Discapacitadas. Informe País. $201 \mathrm{I}$.

14. OMS. Informe Mundial sobre la Discapacidad 20II. Disponible en: www.who.int/disabilities/ world_report/20I l/es/index.html

15. Pantano, Liliana. Discapacidad: conceptualización, magnitud y alcances. (2008) Apuntes para el mejoramiento de las prácticas en relación a las personas con discapacidad Centro de Investigaciones Sociológicas. Departamento de Sociología. FCSE. UCA /CONICET. Documento de trabajo. 